\title{
miR-375 exhibits a more effective tumor-suppressor function in laryngeal squamous carcinoma cells by regulating KLF4 expression compared with simple co-transfection of miR-375 and miR-206
}

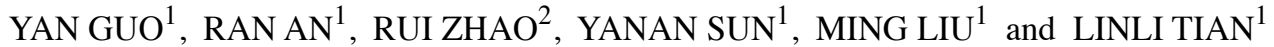 \\ Services of ${ }^{1}$ Head and Neck Surgery, and ${ }^{2}$ Laryngology, Department of Otolaryngology-Head and Neck Surgery, \\ The Second Affiliated Hospital of Harbin Medical University, Harbin, Heilongjiang 150081, P.R. China
}

Received January 12, 2016; Accepted February 26, 2016

DOI: $10.3892 /$ or.2016.4852

\begin{abstract}
MicroRNAs (miRNAs) are reported to be important regulators of cancer-related processes, and function either as oncogenes or as tumor-suppressor genes. It was found that miR-375 was downregulated in samples of laryngeal squamous cell carcinomas (LSCCs) as compared to the level noted in adjacent non-tumor tissues, and it was inversely correlated with $\mathrm{T}$ grade, lymph node metastases and clinical tumor stage. Overexpression of miR-375 led to a decreased protein level of Krüppel-like factor 4 (KLF4) and marked suppression of the proliferation and invasion, and induced apoptosis of LSCC cell line Hep-2 using Cell Counting Kit-8, Transwell chamber and cell cycle assays. In addition, we examined the influence of the upregulation of miR-206 alone and upregulation of both miR-375 and miR-206 on the expression of KLF4 and Hep-2 cell behavior. The results showed that compared with the function of miR-375 in tumor suppression by regulating KLF4, co-transfection of miR-375 and miR-206 exhibited a less effective inhibitory effect not only on tumor cell proliferation and invasion, but also on tumor cell apoptosis. Taken together, miR-375 is possibly a tumor suppressor in LSCC by regulating KLF4. In addition, simple overexpression of several miRNAs did not entail higher efficacy than a single miRNA, similar to co-transfecions of miR-375 and miR-206.
\end{abstract}

Correspondence to: Dr Ming Liu and Dr Linli Tian, Service of Head Neck Surgery, Department of Otolaryngology-Head and Neck Surgery, The Second Affiliated Hospital of Harbin Medical University, 148 Bao Jian Road, Harbin, Heilongjiang 150086, P.R. China

E-mail: liumingorl@qq.com

E-mail: tianlinli78@163.com

Key words: miR-375, miR-206, laryngeal squamous cell carcinoma, cell growth, invasion, co-transfection

\section{Introduction}

MicroRNAs (miRNAs) are a class of non-coding RNAs that are 22 nucleotides in length. Since their discovery in 1993, miRNAs have been shown to play a profound role in the regulation of many aspects of cell function $(1,2)$. miRNAs are often upregulated or downregulated in human types of cancers, and can affect different stages of tumor development to promote or inhibit tumor growth by post-transcriptionally downregulating or suppressing mRNA expression $(3,4)$. In particular, many miRNAs that are expressed at low levels have been demonstrated to play a role in tumor suppression in cancers including laryngeal squamous cell carcinomas (LSCCs).

LSCC, is one of the most common head and neck cancers worldwide and, accounts for nearly $90 \%$ of all malignant laryngeal cancers (5). Despite recent advances in chemoradiotherapy and surgery, there have been no clear improvements in the prognosis of this disease (6). Although the pathology of LSCC has been attributed to many factors, the accumulation of genetic and epigenetic changes remains the most favored basic mechanism of tumorigenesis (7). Recent studies have identified many miRNAs that are abnormally expressed in multiple solid tumors, such as gastric (8), liver (9) and ovarian cancer (10), including LSCC (11). We previously investigated the functions of a series of miRNAs in LSCC. It was found that the expression levels of miR-19a, miR-21, miR-129 and miR-206 were decreased, whereas the expression levels of miR-203 and miR-205 were increased in LSCC (11-17). Functional analyses indicated that these miRNAs, which function as oncogenes or tumor suppressors, have indeed been implicated in the carcinogenesis of LSCC. Recently, studies have shown that synergistic expression of certain miRNAs could jointly effect tumor behavior $(18,19)$. To validate the function of the coordination among miRNAs, the present study aimed to investigate whether the combined expression of miRNAs have superior tumor-suppression effects to those of a single miRNA.

miR-375 is located on human chromosome 2 q35 (20). To date, low levels of miR-375 expression have been detected in prostate (21) and esophageal cancer (22), and other types of cancers. miR-206 is considered to promote muscle differentiation by downregulating the DNA polymerase P180 subunit and 
transcription factors (23). We previously found that miR-206 was downregulated in LSCC. Transfection of miR-206 in Hep-2 cells suppressed tumor proliferation, invasion and induced apoptosis, indicating that miR-206 functions as a novel tumor-suppressor gene in this cancer (13-17).

Krüppel-like factor 4 (KLF4), also known as gut-enriched Krüppel-like factor, was recently identified as a Krüppel-type transcription factor with three $\mathrm{C}_{2} \mathrm{H}_{2}$ zinc fingers (24). KLF4 regulates the phenotype of tumor and stem cells (25), inhibits the formation of tumors in sites such as the gastrointestinal tract (26), and can promote malignant characteristics in other tissues, including breast and skin tissues (27-29). Metacore pathway analysis predicted that KLF4 is a target of miR-206, which was further supported by sequence complementarity alignment (30), and miR-375 directly inhibits the expression of KLF4 by targeting its 3' untranslated region (7).

In the present study, it was found that the expression of miR-375 was downregulated in LSCC tissues. miR-375 was found to inhibit the tumor cell proliferation, migration and invasion, and induce the apoptosis of LSCC cells, by regulating KLF4 as a target gene. These effects were superior to that of the combined expression of miR-375 and miR-206. miR-375 may act as a tumor suppressor and serve as a potential therapeutic target in LSCC.

\section{Materials and methods}

Patients and tissue collection. A retrospective review of 60 adult patients with pathologically confirmed primary LSCC was performed. The use of clinical materials was approved by the local Ethics Committee of the Second Affiliated Hospital of Harbin Medical University. Between 2012 and 2015 , these patients underwent a partial or total laryngectomy at the Department of Otorhinolaryngology and Head and Neck Surgery at The Second Affiliated Hospital of Harbin Medical University. The matched specimens of LSCCs and the corresponding adjacent non-neoplastic tissues obtained from 60 patients were preserved in liquid nitrogen within 5 min after tumor resection, and were then stored at $-80^{\circ} \mathrm{C}$. The study protocol used was in accordance with the Institutional Guidelines for Human Research and was approved by the ethics committee.

Lentiviral vectors for miR-206 and miR-375. Human miR-375 and miR-206 lentivirus gene transfer vectors harboring green fluorescent protein (GFP) sequence were constructed by GeneChem (Shanghai, China): miR-206, 5'-TCCCAGTGAT CTTCTCGCTAAGAGTTTCCTGCCTGGGCAAGGAGGA AAGATGCTACAAGTGGCCCACTTCTGAGATGCGGGC TGCTTCTGGATGACACTGCTTCCCGAGGCCACATGCT TCTTTATATCCCCATATGGATTACTTTGCTATGGAAT GTAAGGAAGTGTGTGGTTTCGGCAAGTGCCTCCTCG CTGGCCCCAGGGTACCACCCGGAGCACAGGTTTGGT GACCTTCTTCCTCATCAGGGCTTTGTGCCAGCAAAT GACTCCCTCACCAAGGAAGTTTTTT-3' and miR-375, 5'-AGGCTAGCGGGGCGCTGTGCAGCACTGAGCTCGC GGAAGACCAGGACCAGGAGATCACCGAGGGCGACC GCCAGGCCCCGGGCCCTCCGCTCCCGCCCCGCGAC GAGCCCCTCGCACAAACCGGACCTGAGCGTTTTGTT CGTTCGGCTCGCGTGAGGCAGGGGCGGCCTCTCAG
CACCAGCCCGGGGGCCGGCCTGATCGCCACGCAGG CACCTGCCGCCGCCA-3'. The recombinant lentiviruses of miR-206 and miR-375, and the control lentivirus (GFP-lentivirus) were prepared and titered to $10^{8} \mathrm{TU}$ (transfection units)/ml, according to the manufacturer's guidelines (GeneChem).

Cell culture and transfections. The human LSCC cell line, Hep-2, was purchased from the Cell Bank of the Chinese Academy of Science (Shanghai, China). Cells were cultured in Dulbecco's modified Eagle's medium (DMEM; HyClone, Logan City, UT, USA) with high glucose, supplemented with $10 \%$ fetal bovine serum (FBS; Shanghai Shenggong Co., Ltd., Shanghai, China) and $1 \%$ penicillin/streptomycin (Beyotime Biotechnology, Shanghai, China) and were maintained at $37^{\circ} \mathrm{C}$ under a humidified atmosphere containing $5 \% \mathrm{CO}_{2}$. Six-well plates were maintained at a concentration of $1 \times 10^{5}$ cells/ well for transfection. After $24 \mathrm{~h}$, when the cells reached $\sim 70-80 \%$ confluency, $1 \mathrm{ml}$ of complete medium containing lentivirus $\left(10^{8} \mathrm{TU} / \mathrm{ml}\right)$ preparations and Polybrene $(5 \mu \mathrm{g} / \mathrm{ml})$ were added to each well. The cells were incubated at $37^{\circ} \mathrm{C}$ for $12 \mathrm{~h}$. The supernatant from the cells was then removed, and DMEM containing $10 \% \mathrm{FBS}$ and $1 \%$ penicillin-streptomycin was added. After $24 \mathrm{~h}$, the culture medium was replaced with fresh DMEM. At $72 \mathrm{~h}$ post-transfection, the mean percentage of GFP-positive cells observed in each well was calculated from three random fields of view at a magnification of x200 using a fluorescence microscope (IX70; Olympus, Tokyo, Japan).

Extraction of total RNA and quantitative real-time polymerase chain reaction. Total RNA was extracted using TRIzol (Invitrogen, Carlsbad, CA, USA) according to the manufacturer's protocol. RNA concentrations were determined using absorbance readings at $260 \mathrm{~nm}$, while RNA purity was evaluated using the optical density $(\mathrm{OD})_{260} / \mathrm{OD}_{280}$ absorption ratios. cDNA was reverse transcribed using an All-in-One ${ }^{\mathrm{TM}}$ miRNA quantitative polymerase chain reaction (qPCR) detection kit (GeneCopoeia, Rockville, MD, USA). The reverse transcription (RT) reactions were incubated at $37^{\circ} \mathrm{C}$ for $60 \mathrm{~min}$ and then at $70^{\circ} \mathrm{C}$ for $5 \mathrm{~min}$. Real-time qPCR was performed using a SYBR-Green Master Mix and a 7500 Fast Real-Time PCR system (both from Applied Biosystems, Carlsbad, CA, USA). Reactions were incubated at $95^{\circ} \mathrm{C}$ for $10 \mathrm{~min}$, followed by 40 cycles at $95^{\circ} \mathrm{C}$ for $10 \mathrm{sec}, 60^{\circ} \mathrm{C}$ for $20 \mathrm{sec}$ and $72^{\circ} \mathrm{C}$ for $30 \mathrm{sec}$. For the measurement of the KLF4 transcript from total RNA, total cDNA was synthesized using ReverTra Ace qPCR RT kit (Toyobo Co., Ltd., Osaka, Japan). Real-time PCR was performed using SYBR Real-Time PCR Master Mix (Toyobo Co., Ltd.). The ubiquitin 6 small nuclear RNA (snRNA) and $\beta$-actin were used as endogenous controls for miRNA and mRNA, respectively. The $\Delta \Delta \mathrm{Ct}$ method was used to determine relative quantitation of miRNA and expression of mRNA in tissue samples, and fold-change was determined as $2^{-\Delta \Delta \mathrm{Ct}}$. The primer sequences of miR-375 (product code, CD201-0173) and endogenous control U6 (CD201-01730145) were applied by Tiangen Biotech (Beijing, China). KLF4, 5'-CTTCCTGCCC GATCAGATGC-3' and 5'-TCGCAGGTGTGCCTTGAGT-3'; $\beta$-actin, 5'-CTTAGTTGCGTTACACCCTTTCTTG-3' and 5'-CTGTCACCTTCACCGTTCCAGTTT-3' were from 
Applied Bioneer (Daejeon, Korea). Each reaction was performed in triplicate.

Western blot analysis. At $72 \mathrm{~h}$ post-transfection with miR-375 and miR-206, the cells were subjected to western blot analysis. Cells were incubated in cell lysis buffer for $30 \mathrm{~min}$ on ice. Cell lysates were separated using $10 \%$ sodium dodecyl sulfatepolyacrylamide gel electrophoresis and were then transferred to polyvinylidene fluoride membranes. After incubating the membranes in 5\% skim milk in Tris-buffered saline containing $0.05 \%$ Tween-20 (TBST), the membranes were incubated with primary antibodies overnight at $4^{\circ} \mathrm{C}$. The primary antibodies used for western blotting included anti-KLF4 (1:1,000; Cell Signaling Technology, Boston, MA, USA). The membranes were then washed with TBST and incubated with species-appropriate horseradish peroxidase-conjugated secondary antibodies for $1 \mathrm{~h}$ at $37^{\circ} \mathrm{C}$. $\beta$-actin $(1: 5,000$; Bioworld Technology, Inc., St. Louis Park, MN, USA) served as a loading control, and bands were quantified using ImageJ software (National Institutes of Health, Bethesda, MD, USA). Three independent experiments were performed.

Cell proliferation assay. After transfection of Hep-2 cells by lentivirus for varying durations of time: $12,24,48$ and $72 \mathrm{~h}$, $100 \mu 1$ of sterile Cell Counting Kit-8 [2-(2-methoxy-4-nitrophenyl)-3-(4-nitrophenyl)-5-(2,4-disulfophenyl)-2H-tetrazolium monosodium salt] (Dojindo Co., Ltd., Shanghai, China) was added and incubated for another $4 \mathrm{~h}$ at $37^{\circ} \mathrm{C}$. Later, spectrometric absorbance at a wavelength of $450 \mathrm{~nm}$ was measured on an enzyme immunoassay analyzer (model 680; Bio-Rad Laboratories, Hercules, CA, USA). The rate of cell growth was calculated using the following formula: Cell growth rate $(\%)=$ (mean absorbance in 96-wells of the treatment group/mean absorbance in 6-wells of the cells in the control group) $\mathrm{x} 100$.

Transwell chamber invasion assay. Cells were washed, resuspended in complete RPMI-1640 medium ( $\left.1 \times 10^{5} \mathrm{cells} / \mathrm{ml}\right)$, and added to the upper chamber of Boyden chambers (24-well, $8-\mu \mathrm{m}$ pores) coated with Matrigel (Becton-Dickinson Labware, Franklin Lakes, NJ, USA). The lower chamber contained $600 \mu \mathrm{l}$ conditioned medium. After $24 \mathrm{~h}$ of incubation, the inserts and cells were mechanically removed from the upper side of the filters. Filters were fixed in $4 \%$ paraformaldehyde and stained with hematoxylin and eosin. Cells were observed on a microscope, and five randomly selected fields were observed at a magnification of $x 400$. Tests were repeated in triplicate.

Cell cycle assay. Cells were fixed with cold ethanol at $4^{\circ} \mathrm{C}$ for $1 \mathrm{~h}$, and then stored at $-20^{\circ} \mathrm{C}$ until analysis. Cells were washed with phosphate-buffered saline (PBS), treated with RNase A $(50 \mu \mathrm{g} / \mathrm{ml})$ and stained with ethidium bromide for $20 \mathrm{~min}$ at $37^{\circ} \mathrm{C}$. Cells were analyzed for DNA content using flow cytometry (FACSCalibur; Becton-Dickinson Immunocytometry Systems, San Jose, CA, USA) and the distribution of cell cycle phases was determined using ModFit LT for Mac V 3.0.

Apoptosis assay. The Annexin V-fluorescein isothiocyanate (FITC) and propidium iodide (PI) double staining detection
Table I. Correlation between the miR-375 expression level and the clinicopathological parameters of the LSCC patients.

miR-375

Characteristics

n (T/N expression ratio) P-value

Gender

Male

Female

52

$0.224 \pm 0.006$

$8 \quad 0.228 \pm 0.003$

Age (years)

$\geq 60$

$<60$

27

$0.245 \pm 0.010$

$33 \quad 0.239 \pm 0.004$

$\mathrm{T}$ classification

T1-2

38

$0.251 \pm 0.007$

T3-4

22

$0.227 \pm 0.003$

Lymph node metastasis

Negative

Positive

$35 \quad 0.251 \pm 0.008$

$25 \quad 0.230 \pm 0.003$

Primary location

Supraglottic

Glottic

31

29

$0.241 \pm 0.009$

$0.243 \pm 0.003$

Clinical stage

I-II

34

$0.254 \pm 0.008$

III-IV

26

$0.226 \pm 0.002$

$\mathrm{T} / \mathrm{N}$ expression ratio, tumor/non-cancerous tissue expression ratio: the fold-change in miR-375 levels relative to the human U6 gene in tumor and matched normal tissues according to the $2^{-\Delta A C t}$ method. Values are expressed as the means \pm SEM for each group. T/N, tumor/non-cancerous tissues.

kit was used to measure apoptosis according to the manufacturer's protocol (Wanleibio, Shenyang, China). The cells were resuspended in binding buffer (Beyotime Biotechnology) at a concentration of $1 \times 10^{6}$ cells $/ \mathrm{ml}$. The cells were incubated with $5 \mu 1$ Annexin V-FITC, stained with $5 \mu 1$ PI, and then incubated in the dark at room temperature for $15 \mathrm{~min}$. Cells were analyzed using flow cytometry within $1 \mathrm{~h}$.

Statistical analyses. Data are expressed as the means \pm SEM of three independent experiments, each performed in triplicate. Statistical significance was tested using SPSS 19.0 software. An independent t-test was used to analyze differences in KLF4 mRNA levels after transfection, KLF4 protein scoring, cell viability, invasive phenotype, apoptosis induction and cell cycle distribution between groups. For analysis of differences in miR-375 mRNA and KLF4 mRNA between tumor samples and adjacent normal tissues, the data were checked by paired $\mathrm{t}$-test. P-value $<0.05$ was considered to indicate a statistically significant result.

\section{Results}

miR-375 is downregulated in human LSCCs. We previously reported that the expression of miR-206 is decreased in LSCCs (13). In the present study, the expression of miR-375 
A
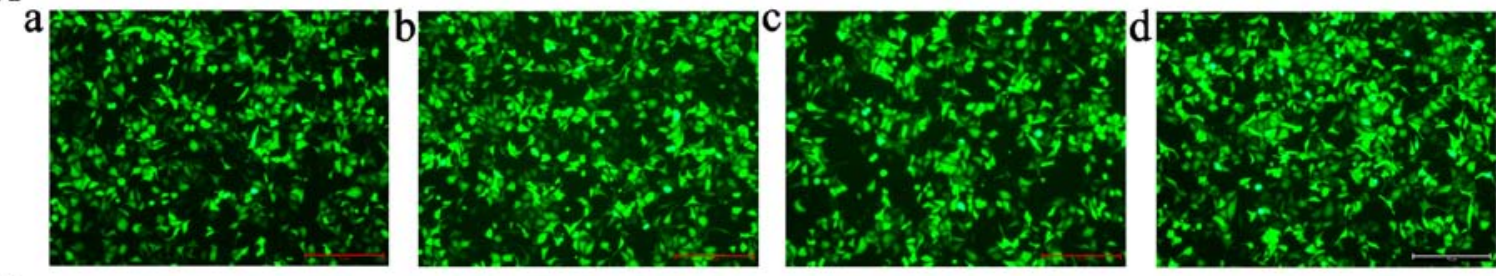

$\mathrm{B}$
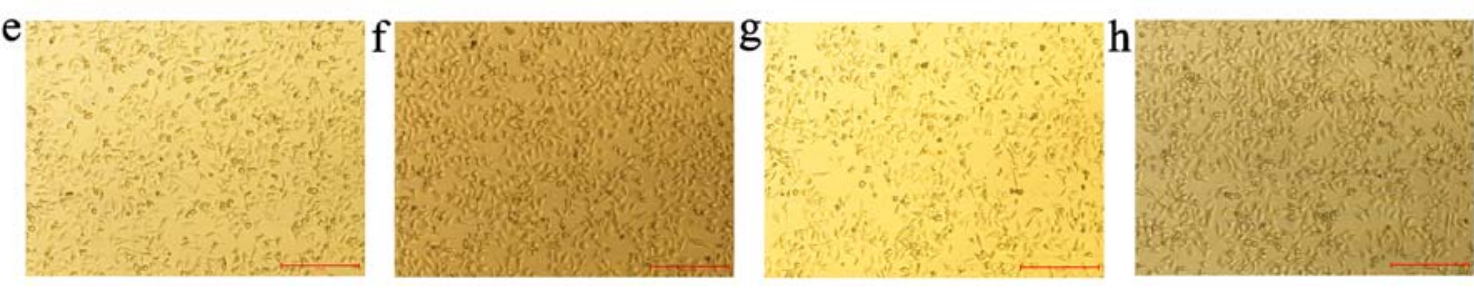

Figure 1. (A) Fluorescence microscopic images of (a) GFP-transfected control group; (b) miR-375-transfected Hep-2 cells; (c) miR-206-transfected Hep-2 cells and (d) miR-375 + miR-206-transfected Hep-2 cells. (B) Light microscopy images of (e) GFP-transfected control group; (f) miR-375-transfected Hep-2 cells; (g) miR-206-transfected Hep-2 cells and (h) miR-375 + miR-206-transfected Hep-2 cells. GFP, green fluorescence protein.

was assessed in 60 patients. The clinicopathological findings of the patients are shown in Table I. To determine the expression of miR-375 in LSCC, qRT-PCR was used to assess the expression levels of RNA in the LSCC tissue samples and adjacent normal tissues collected from the same 60 patients. Similar to miR-206, the expression of miR-375 was also decreased in the tumor samples $(0.524 \pm 0.009)$, when compared with that in the adjacent non-cancerous tissues $(2.179 \pm 0.019)(\mathrm{P}<0.01)$. The tumor/non-cancerous tissue $(\mathrm{T} / \mathrm{N})$ ratios for the expression of miR-375 were found to be significantly correlated with $\mathrm{T}$ stage, tumor differentiation, neck nodal metastasis and clinical stage. Decreased expression of miR-375 was found in the tumors from patients with advanced clinical stage, T3-4 grade or lymph node metastasis.

Transfection of miR-375 and miR-206 inhibits cell viability, respectively. To investigate the biological function of miR-375 and miR-206 in LSCC, recombinant lentiviruses containing miR-375 and miR-206 mimics as well as a GFP cassette, were created. At $72 \mathrm{~h}$ after Hep-2 cells were transfected with miRNAs and GFP control lentiviruses, $>80 \%$ of the Hep-2 cells were found to express GFP, indicating the efficiency and stability of the transductions (Fig. 1). The expression of miRNAs in the different experimental groups was then confirmed using qRT-PCR (data not shown).

All the experimental cells in the present study were categorized into five groups: blank control group that contained untreated Hep-2 cells; GFP control group that contained Hep-2 cells transfected with lentiviruses without miRNAs; miR-375 group that contained Hep-2 cells transfected with lentiviruses with miR-375 mimics; miR-206 group that contained Hep-2 cells transfected with lentiviruses with miR-206 mimics; and miR-375 + miR-206 group that contained Hep-2 cells transfected with lentiviruses with miR-375 and miR-206 mimics.

As shown in Fig. 2 after transfection with the miRNAs, the proliferation rates of the Hep-2 cells in the GFP control group did not show any obvious alteration during the time course. However, the viability of the miR-375 group Hep-2 cells was evidently decreased at each time point $(12,24,48$ and $72 \mathrm{~h}$ ). The viability of the miR-206 group Hep- 2 cells was

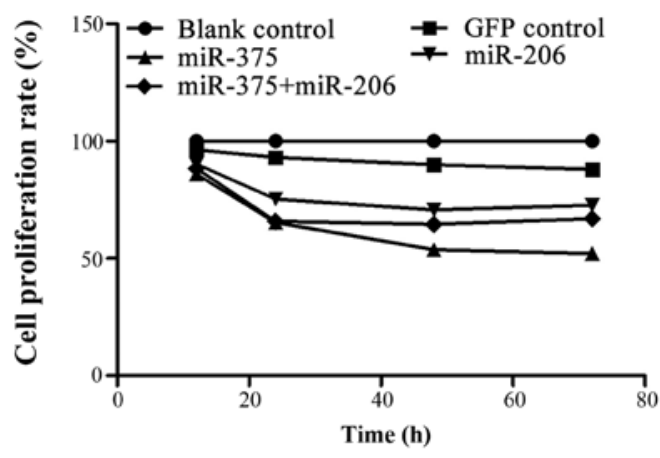

Figure 2. Curve showing the rate of cell proliferation. After transduction with miR-375 and miR-206 lentivirus, the rate of proliferation of Hep-2 cells was lower at each time points $(12,24,48$ and $72 \mathrm{~h}$, respectively) compared with the controls.

also decreased compared with the blank control group, but the proliferation rate curve had a smaller decrease after $24 \mathrm{~h}$, and its downward trend was less pronounced than that of miR-375. Additionally, an initial evident decrease in the survival rate curve of the miR-375 + miR-206 group was noted, which was similar to the miR-375 group. This was followed by a relative stable state after $24 \mathrm{~h}$, and its downward trend was always between the levels of the miR-375 and miR-206 groups. These findings indicated that reconstitution of miR-375, miR-206 or both miRNAs could inhibit the viability of Hep-2 cells in vitro. Furthermore, transfection with miR-375 alone was found to be the most effective.

Overexpression of miR-375 and miR-206 promotes early apoptosis in Hep-2 cells. Flow cytometric analysis revealed that transfection of Hep-2 cells with miRNAs for $72 \mathrm{~h}$ significantly induced higher levels of apoptosis, compared with cells of the GFP control or the uninfected cells. As shown in Fig. 3 the apoptosis rate of the GFP control group (2.8\%) and blank control group cells $(2.6 \%)$ were nearly the same. However, the apoptosis rate of miR-375 group cells (67.2\%) was much higher than the blank control group cells. Compared with the blank 

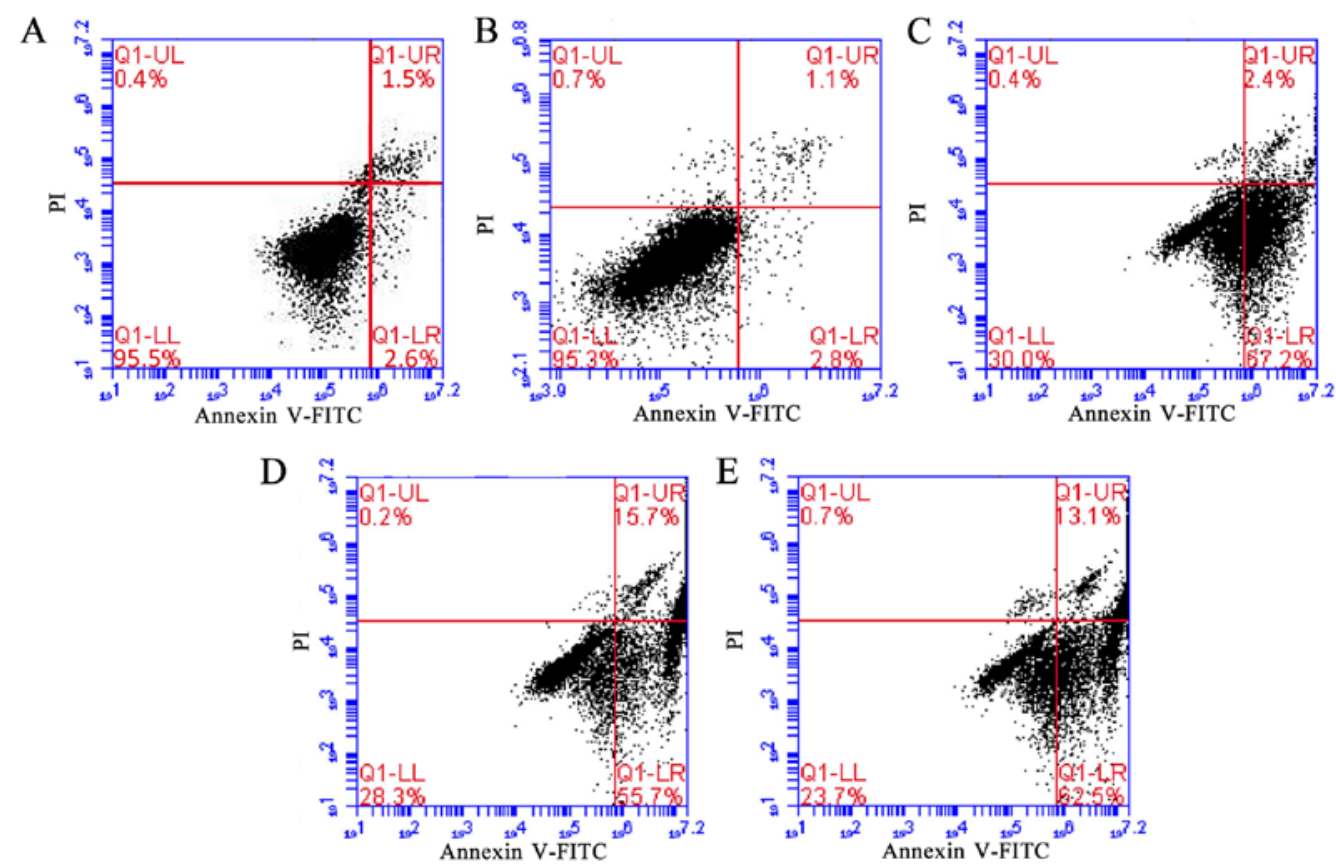

Figure 3. After transfection for $72 \mathrm{~h}$, miRNAs induced Hep-2 cell apoptosis. The dot plot of the $\mathrm{x}$-axis shows the log of Annexin V-FITC fluorescence and the $y$-axis shows PI fluorescence, obtained by flow cytometry. (A) Representative image of Hep-2 cells without any treatment. (B) Representative image of Hep-2 cells infected with GFP-lentivirus control. (C) Representative image of Hep-2 cells infected with the miR-375 lentivirus. (D) Representative image of Hep-2 cells infected with miR-206. (E) Representative image of Hep-2 cells infected with miR-375 + miR-206. FITC, fluorescein isothiocyanate; GFP, green fluorescence protein; miRNA, microRNA; PI, propidium iodide.
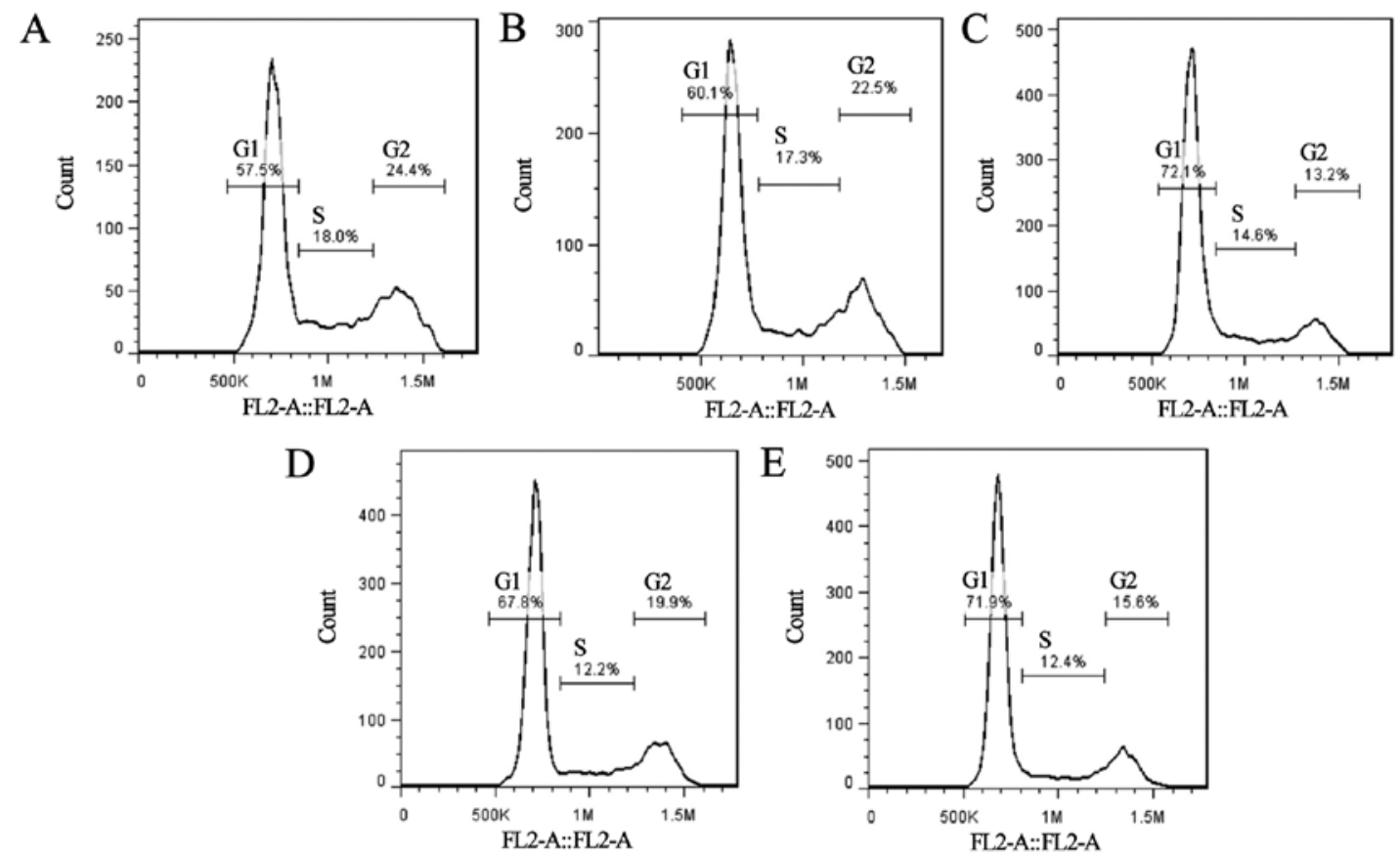

Figure 4. Flow cytometric analysis of the effect of miRNAs on the cell cycle distribution in Hep-2 cells after transfection for $72 \mathrm{~h}$. (A) Cell cycle analysis of the blank control group Hep-2 cells. (B) Cell cycle analysis of GFP control group Hep-2 cells. (C) Cell cycle analysis of miR-375-transfected Hep-2 cells. (D) Cell cycle analysis of miR-206-transfected Hep-2 cells. (E) Cell cycle analysis of miR-375 + miR-206 Hep-2 cells-transfected Hep-2 cells. GFP, green fluorescence protein; miRNA, microRNA.

group, the apoptosis rate of miR-206 group cells (55.7\%) was also apparently higher than that noted in the untreated cells, but lower than that noted in the miR-375 group. The apoptosis rate of co-transfection group cells $(62.5 \%)$ was between the rated observed in the miR-375 and miR-206 group. These results confirmed the strong pro-apoptotic effect of miR-375 and miR-206, however, the effect of miR-375 on the promotion of apoptosis was stronger than the effect by miR-206, and this effect was even stronger than miR-375 combined with miR-206. 

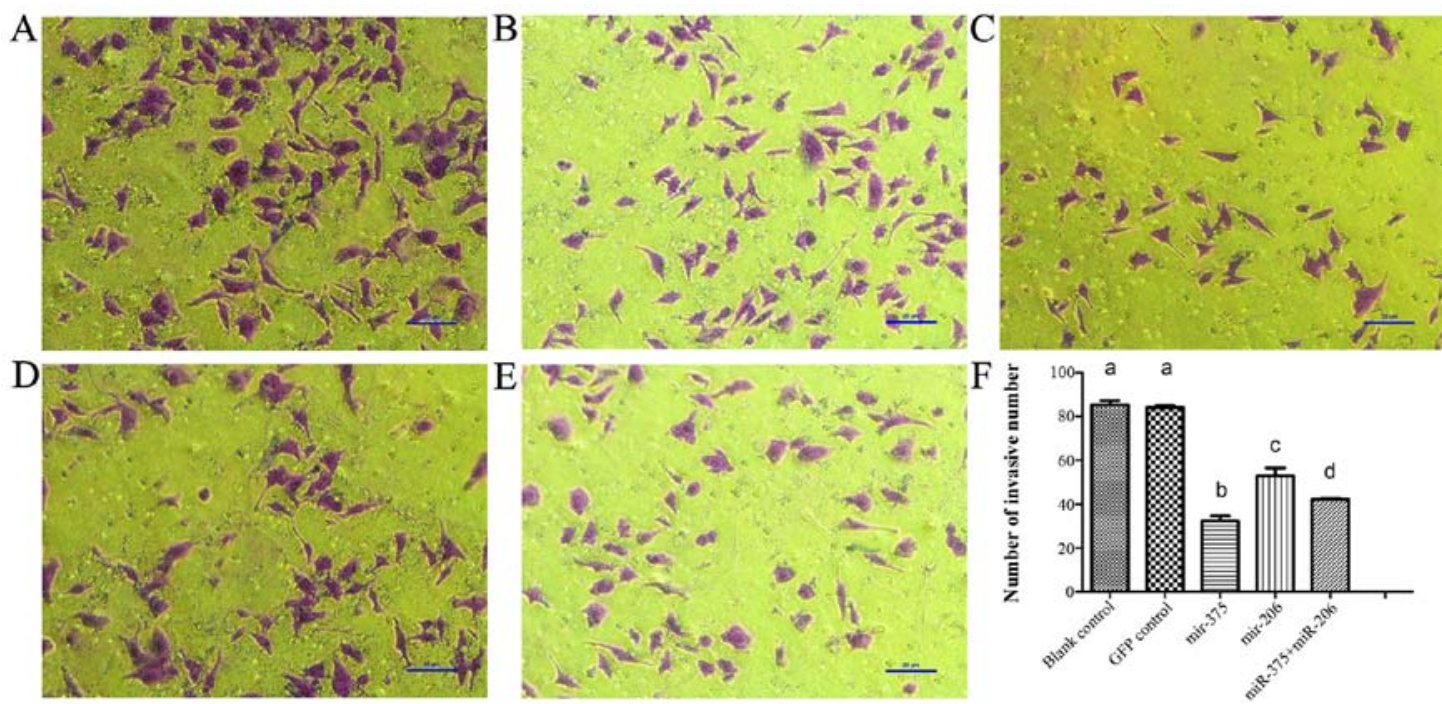

Figure 5. Invasive phenotype of the lentivirus-transfected cells. (A) Representative image of migrating cells in the untreated group after $72 \mathrm{~h}$ of transfection. (B) Representative image of migrating cells in the GFP group after $72 \mathrm{~h}$ of transfection. (C) Representative image of migrating cells in the miR-375 group after $72 \mathrm{~h}$ of transfection. (D) Representative image of migrating cells in the miR-206 group after $72 \mathrm{~h}$ of transfection. (E) Representative image of migrating cells in the miR-206 + miR-375 group after $72 \mathrm{~h}$ of transfection. (F) The mean number of Hep-2 cells in each group that migrated to the lower chambers of the Transwell plates is shown. (The different lowercase letters above the columns indicate significant differences between the groups; $\mathrm{P}<0.01$ ).
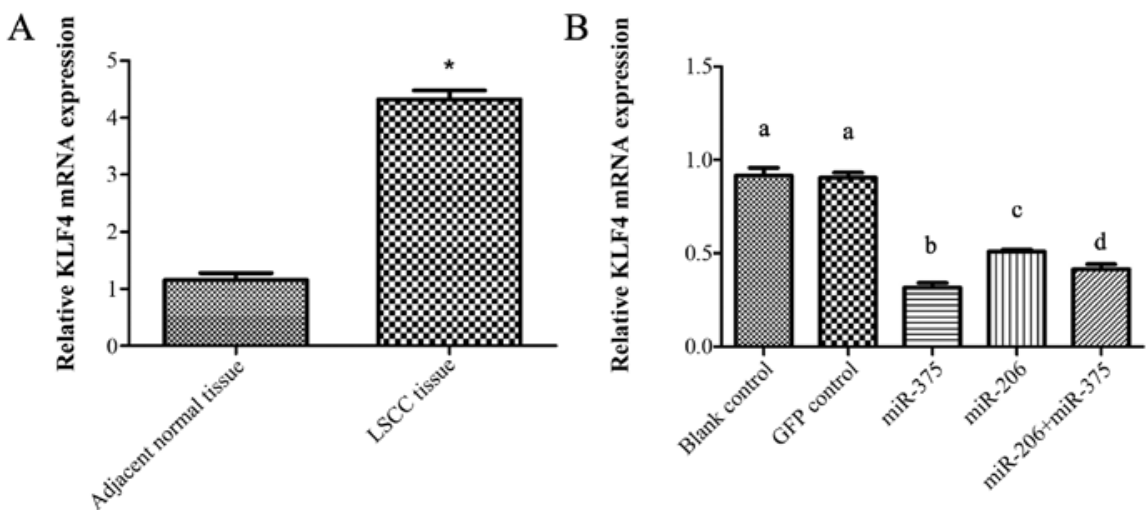

Figure 6. (A) Expression of KLF4 mRNA in LSCC and adjacent normal tissues. KLF4 mRNA levels were 3.91-fold higher in the tumor tissues compared with that in the adjacent normal tissues as detected by real-time RT-PCR ("P<0.05). (B) After transfection for $72 \mathrm{~h}, \mathrm{Hep}-2$ cells transfected separately with miR-375, miR-206 and miR-375 + miR-206 lentivirus exhibited a significantly lower KLF4 mRNA level than that noted in the Hep-2 cells transfected with a GFPcontrol lentivirus or the untreated Hep-2 cells, according to real-time RT-PCR assays. (The different lowercase letters above the columns indicate significant differences between the groups; P<0.01). GFP, green fluorescence protein; KLF4, Krüppel-like factor; LSCC, laryngeal squamous cell carcinoma; RT-PCR, reverse transcription-polymerase chain reaction.

miR-375 and miR-206 overexpression affects cell cycle progression in the Hep-2 cells. miR-375-transfected Hep-2 cells $(72.1 \%)$ showed the highest percentage of cells in the G1 phase compared to the GFP control cells $(60.1 \%)$ and blank control cells $(57.5 \%)$ at $72 \mathrm{~h}$ post-transfection $(\mathrm{P}<0.05)$. This percentage in the miR-206-transfected cells $(67.8 \%)$ was higher than that noted in the GFP control cells or untreated group cells, but lower than that in the miR-375 group cells. The percentage of cells arrested in the G1 phase in the miR-375 + miR-206-transfected cells (71.9\%) was between the percentage of cells in the miR-375 and miR-206 groups (Fig. 4). This demonstrates that miR-375 and miR-206 upregulation can both produce G1 phase arrest since the number of cells in the G1 phase increased by $>10 \%$ when compared with the control groups. In addition, an increase in miR-375 alone had the strongest effect on the G1 phase arrest.
miR-375 and miR-206 overexpression suppresses Hep-2 cell invasion in vitro. To investigate whether miR-375 and miR-206 overexpression is beneficial to the Hep-2 cell invasive phenotype, invasion assays were performed using 24-well Boyden chambers coated with Matrigel. The number of Hep-2 cells that passed through the filter after transfection with miRNAs for $72 \mathrm{~h}$ was much lower than that observed for the GFP-control group cells $(84 \pm 1.15)$ and blank control cells (87 \pm 2.03$)$. Among the three miRNA-transfected groups, cells that were transfected with miR-375 $(32.3 \pm 2.33)$ showed less robust invasion than the group transfected with miR-206 $(53 \pm 3.51)$ and miR-375 + miR-206 $(42 \pm 0.33)$. The number of invasive cells of the miR-206 group was higher than that noted in the miR-375 group as well as the co-transfection group. In addition, the number of invasive cells in the co-transfection group was between this number noted in the miR-375 and 

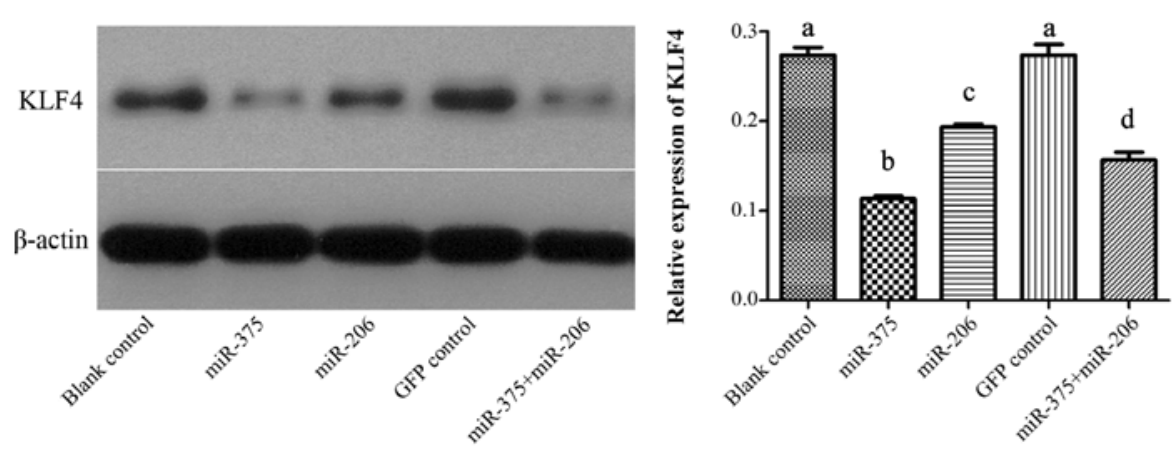

Figure 7. Downregulation of KLF4 protein levels in Hep-2 cells transfected with miR-375, miR-206 or miR-375 + miR-206 lentivirus results in lower levels of KLF4 protein expression as compared with cells transfected with a GFP-control lentivirus or untransfected cells. (The different lowercase letters above the columns indicate significant differences between the groups; P<0.01). GFP, green fluorescence protein; KLF4, Krüppel-like factor.

miR-206 groups (Fig. 5). However, the inhibitory effect on invasion by miR-375 was stronger than that of miR-206 and miR-375 + miR-206, and the inhibitory effect of miR-206 was the weakest.

Overexpression of miR-375 and miR-206 suppresses the expression of KLF4. KLF4 is an important gene with effective transcriptional regulation on various downstream genes involved in cell cycle, apoptosis, proliferation and invasion. To compare the expression of KLF4 in LSCC and adjacent normal tissues, KLF4 mRNA in 60 patients with LSCC samples and the corresponding adjacent non-neoplastic tissues were performed using qRT-PCR. As shown in Fig. 6A, the expression level of KLF4 mRNA in LSCC was $\sim 4$-fold higher than that of the corresponding matched samples.

To detect the expression of KLF4 following regulation of miR-375 and miR-206 in the Hep-2 cells after transfection, KLF4 mRNA and protein expression was examined using qRT-PCR and western blotting, respectively.

As shown in Fig. 6B, after transfection, the level of KLF4 mRNA in the GFP control group did not show an obvious change as compared with the blank control group. However, the KLF4 mRNA level in the miR-375 group showed a much lower level than that in the blank control group. Expression of KLF4 mRNA in the miR-206 group was also lower than that in the blank control group, but higher than that in the miR-375 group. In addition, the KLF4 mRNA level in the miR-375 + miR-206 group was lower than that in the miR-206 group but higher than that in the miR-375 group $(\mathrm{P}<0.05)$.

Results of western blotting showed that the expression of KLF4 protein was significantly downregulated after forced expression of miR-375. The level of KLF4 in the miR-206 group was also decreased compared with the control groups, but not as much as miR-375. In addition, the expression of KLF4 in the co-transfection group was lower than that in the miR-206 group but higher than that in the miR-375 group $(\mathrm{P}<0.05)$. However, cells in the GFP control group did not show any significant changes in the expression of KLF4 protein as compared to the untreated Hep-2 cells ( $\mathrm{P}>0.05$; Fig. 7).

\section{Discussion}

Increasing evidence suggests that miRNAs play key roles in diverse biological processes, including development, cell proliferation, differentiation and apoptosis $(31,32)$. Furthermore, miRNAs have also been reported to play essential roles in carcinogenesis and tumor progression $(33,34)$. Numerous published studies have reported that miR-375 is downregulated in several types of cancer $(35,36)$. In the present study, it was confirmed that miR-375 was downregulated in LSCC, which is consistent with the results of Luo et al (37). It was found that lower expression of miR-375 was closely correlated with lymph node metastasis, advanced clinical stage and high $\mathrm{T}$ classification. Considering the association of these clinicopathological parameters with the poor prognosis of patients with LSCC, these results imply that miR-375 may play a role in the progression and influence the prognosis in LSCC. To explore the effect of the overexpression of miR-375 on the metastasis and progression of LSCC, we examined the alterations in cell growth and behavior following miR-375 overexpression in the Hep-2 cells. Elevated expression of miR-375 suppressed proliferation, induced cell cycle arrest in the G1 phase, and suppressed cell migration and invasion in the Hep-2 cells. Taken together, these results suggest that miR-375 is a tumor-suppressor in the growth and progression of LSCC. Various previous studies have proposed that the co-regulation of one target gene or even an entire biological module by combinations of miRNAs may lead to potent synergistic effects $(38,39)$. In the present study, both miR-375 and miR-206 were overexpressed to test the effects on tumor cell function. However, the results indicated that the inhibitory effect in the co-infected LSCC cells was not stronger than that noted in the LSCC cells transfected with miR-375 alone, although it was better than that in the Hep-2 cells transfected with miR-206 alone.

KLF4 was of particular interest to us due to its role in proliferation during tumorigenesis and its dual functions in the context of the tumor microenvironment. For instance, KLF4 appears to suppress the formation of tumors in tissues such as the gut (26), while it can promote malignant properties in other tissues, such as the breast and skin (27-29). KLF4 plays an important role in regulating multiple biological functions, including proliferation, survival, epithelial-mesenchymal transition, migration, invasion and capillary tube formation $(40,41)$. Consistent with Suer et al (42), KLF4 was found to be overexpressed in LSCC tumor tissues compared with its level in adjacent normal tissues. In the present study, an inverse correlation was found between the expression of miR-375 
and KLF4 in the LSCC tissues. Furthermore, expression of KLF4 at both the mRNA and protein levels was significantly downregulated by overexpression of miR-375 using miR-375 mimic transfection. KLF4 was also inhibited following overexpression of miR-206, but the inhibitory effect of miR-375 on KLF4 was more obvious than that of miR-375 + miR-206, and the inhibitory effect of miR-206 was the weakest. Taken together, these results suggest that decreased KLF4, induced by restoring miR-375, participates in the progression of LSCC.

Previous studies have indicated that KLF4 can upregulate the expression of miR-206, and the latter can also promote the expression of KLF4 by an autoregulatory feedback loop formed by KLF4 and miR-206 (25), the regulatory mechanism related to tumor initiation (43). Thus, while both miR-375 and miR-206 can act on KLF4 $(7,30)$, the regulatory pathways are different. Furthermore, the two miRNAs may depend on other crucial target genes which participate in the mechanisms of blockade of proliferation of LSCC cells or increase in apoptosis. Hence, no synergistic effects were noted in the LSCC cells by simple co-transfection of miR-375 and miR-206.

Another possible reason concerning the results of the present study is as follows. KLF4 can bind to $\beta$-catenin and decrease mRNA levels, and can also inhibit $\beta$-catenin function and signaling. Furthermore, KLF4 shows inhibitory activity in $\beta$-catenin-mutant cells (44). $\beta$-catenin is overexpressed in LSCC (45). Downregulation of miR-375 is reported to be associated with $\beta$-catenin mutants, suggesting that $\beta$-catenin signaling can repress miR-375 $(46,47)$. It was demonstrated that miR-375 suppresses the expression of KLF4. Thus, KLF4, $\beta$-catenin and miR-375 may mutually restrict only to form a circle. When both miR-375 and miR-206 are overexpressed, exogenous miR-206 may decrease KLF4 mRNA levels, thereby disrupting the mutual restriction relationship among miR-375, KLF4 and $\beta$-catenin, ultimately impairing the inhibitory function of miR-375 toward KLF4, such that the combination of miR-375 and miR-206 did not strengthen the inhibitory effect of miR-375. Such interaction could partially explain the finding that the co-transfection did not have the strongest effect in LSCC cells.

In conclusion, the expression of miR-375 was downregulated in LSCC tissues and was correlated with neck lymph node metastasis, clinical stage and other poor prognostic clinicopathological parameters. The increased expression of miR-375 suppressed cell proliferation, invasion and promoted cell apoptosis of LSCC cells through KLF4 in vitro. Taken together, these results suggest that the overexpression of miR-375 induced the decrease in the expression of target gene KLF4, and consequently inhibited cell malignant behaviors of LSCC. These results also suggest that the miRNA-regulatory network is further complicated by the fact that any single miRNA can regulate hundreds of targets, while multiple miRNAs may converge to control the same process (48).

Unfortunately, the complete mechanisms by which miRNAs regulate the function of KLF4 in LSCC and the doses of miRNAs to KLF4 are not known yet, but the present findings clearly indicate that simply combining several miRNAs with a common target gene does not always yield additive effects. miR-375 presents an exciting new opportunity for the treatment of laryngeal cancer in the future. In addition, assessing the effects of combinatorial miRNA treatment on different tumor types remains necessary, as this information could be useful for developing therapeutic agents targeting LSCC.

\section{Acknowledgements}

The present study was supported by grants from the Natural Science Foundation of China (nos. 81402234, 81572647 and 81372902), the National Science Foundation of China, and the National Science Foundation of Heilongjiang Province (QC2013C117, ZD201215/H1302).

\section{References}

1. Lee RC, Feinbaum RL and Ambros V: The C.elegans heterochronic gene lin-4 encodes small RNAs with antisense complementarity to lin-14. Cell 75: 843-854, 1993.

2. Iorio MV and Croce CM: MicroRNA dysregulation in cancer: Diagnostics, monitoring and therapeutics. A comprehensive review. EMBO Mol Med 4: 143-159, 2012.

3. Parasramka MA, Ho E, Williams DE and Dashwood RH: MicroRNAs, diet, and cancer: New mechanistic insights on the epigenetic actions of phytochemicals. Mol Carcinog 51: 213-230, 2012.

4. Guo X, Chen Y, Xu Z, Xu Z, Qian Y and Yu X: Prognostic significance of VEGF-C expression in correlation with COX-2, lymphatic microvessel density, and clinicopathologic characteristics in human non-small cell lung cancer. Acta Biochim Biophys Sin 41: 217-222, 2009.

5. Genden EM, Ferlito A, Silver CE, Jacobson AS, Werner JA, Suárez C, Leemans CR, Bradley PJ and Rinaldo A: Evolution of the management of laryngeal cancer. Oral Oncol 43: 431-439, 2007.

6. Ma J, Liu Y, Huang XL, Zhang ZY, Myers JN, Neskey DM and Zhong LP: Induction chemotherapy decreases the rate of distant metastasis in patients with head and neck squamous cell carcinoma but does not improve survival or locoregional control: A meta-analysis. Oral Oncol 48: 1076-1084, 2012.

7. Mao Q, Quan T, Luo B, Guo X, Liu L and Zheng Q: miR-375 targets KLF4 and impacts the proliferation of colorectal carcinoma. Tumour Biol 37: 463-471, 2015.

8. Tsukamoto Y, Nakada C, Noguchi T, Tanigawa M, Nguyen LT, Uchida T, Hijiya N, Matsuura K, Fujioka T, Seto M, et al: MicroRNA-375 is downregulated in gastric carcinomas and regulates cell survival by targeting PDK1 and 14-3-3zeta. Cancer Res 70: 2339-2349, 2010.

9. Ladeiro Y, Couchy G, Balabaud C, Bioulac-Sage P, Pelletier L, Rebouissou $S$ and Zucman-Rossi J: MicroRNA profiling in hepatocellular tumors is associated with clinical features and oncogene/tumor suppressor gene mutations. Hepatology 47: 1955-1963, 2008.

10. Kong KL, Kwong DL, Chan TH, Law SY, Chen L, Li Y, Qin YR and Guan XY: MicroRNA-375 inhibits tumour growth and metastasis in oesophageal squamous cell carcinoma through repressing insulin-like growth factor 1 receptor. Gut 61: 33-42, 2012.

11. Li M, Tian L, Wang L, Yao H, Zhang J, Lu J, Sun Y, Gao X, Xiao H and Liu M: Down-regulation of miR-129-5p inhibits growth and induces apoptosis in laryngeal squamous cell carcinoma by targeting APC. PLoS One 8: e77829, 2013.

12. Ren J, Zhu D, Liu M, Sun Y and Tian L: Downregulation of miR-21 modulates Ras expression to promote apoptosis and suppress invasion of laryngeal squamous cell carcinoma. Eur J Cancer 46: 3409-3416, 2010.

13. Zhang T, Liu M, Wang C, Lin C, Sun Y and Jin D: Downregulation of miR-206 promotes proliferation and invasion of laryngeal cancer by regulating VEGF expression. Anticancer Res 31: 3859-3863, 2011.

14. Tian L, Li M, Ge J, Guo Y, Sun Y, Liu M and Xiao H: miR-203 is downregulated in laryngeal squamous cell carcinoma and can suppress proliferation and induce apoptosis of tumours. Tumour Biol 35: 5953-5963, 2014.

15. Tian L, Zhang J, Ge J, Xiao H, Lu J, Fu S, Liu M and Sun Y: MicroRNA-205 suppresses proliferation and promotes apoptosis in laryngeal squamous cell carcinoma. Med Oncol 31: 785, 2014. 
16. Wu TY, Zhang TH, Qu LM, Feng JP, Tian LL, Zhang BH, Li DD, Sun YN and Liu M: MiR-19a is correlated with prognosis and apoptosis of laryngeal squamous cell carcinoma by regulating TIMP-2 expression. Int J Clin Exp Pathol 7: 56-63, 2013.

17. Ren J, Sun Y, Zhao X, Wang X, Feng J, Liu M and Zhu D: Downregulation of miR-21 regulates MMP-2 expression and suppress migration of Laryngeal squamous cell carcinoma. Head Neck Oncol 4: 65-69, 2012

18. Boll K, Reiche K, Kasack K, Mörbt N, Kretzschmar AK, Tomm JM, Verhaegh G, Schalken J, von Bergen M, Horn F, et al MiR-130a, miR-203 and miR-205 jointly repress key oncogenic pathways and are downregulated in prostate carcinoma. Oncogene 32: 277-285, 2013

19. Kim SY, Lee YH and Bae YS: MiR-186, miR-216b, miR-337-3p, and miR-760 cooperatively induce cellular senescence by targeting $\alpha$ subunit of protein kinase CKII in human colorectal cancer cells. Biochem Biophys Res Commun 429: 173-179, 2012.

20. Baroukh NN and Van Obberghen E: Function of microRNA-375 and microRNA-124a in pancreas and brain. FEBS J 276 : 6509-6521, 2009.

21. Kachakova D, Mitkova A, Popov E, Popov I, Vlahova A, Dikov T, Christova S, Mitev V, Slavov C and Kaneva R: Combinations of serum prostate-specific antigen and plasma expression levels of let-7c, miR-30c, miR-141, and miR-375 as potential better diagnostic biomarkers for prostate cancer. DNA Cell Biol 34: 189-200, 2015

22. Fu C, Dong W, Wang Z, Li H, Qin Q and Li B: The expression of miR-21 and miR-375 predict prognosis of esophageal cancer Biochem Biophys Res Commun 446: 1197-1203, 2014.

23. Kim HK, Lee YS, Sivaprasad U, Malhotra A and Dutta A Muscle-specific microRNA miR-206 promotes muscle differentiation. J Cell Biol 174: 677-687, 2006

24. Geiman DE, Ton-That H, Johnson JM and Yang VW: Transactivation and growth suppression by the gut-enriched Krüppel-like factor (Krüppel-like factor 4) are dependent on acidic amino acid residues and protein-protein interaction. Nucleic Acids Res 28: 1106-1113, 2000.

25. Lin CC, Liu LZ, Addison JB, Wonderlin WF, Ivanov AV and Ruppert JM: A KLF4-miRNA-206 autoregulatory feedback loop can promote or inhibit protein translation depending upon cell context. Mol Cell Biol 31: 2513-2527, 2011.

26. Ghaleb AM, McConnell BB, Nandan MO, Katz JP, Kaestner KH and Yang VW: Haploinsufficiency of Krüppel-like factor 4 promotes adenomatous polyposis coli dependent intestinal tumorigenesis. Cancer Res 67: 7147-7154, 2007.

27. Foster KW, Ren S, Louro ID, Lobo-Ruppert SM, McKie-Bell P, Grizzle W, Hayes MR, Broker TR, Chow LT and Ruppert JM: Oncogene expression cloning by retroviral transduction of adenovirus E1A-immortalized rat kidney RK3E cells: Transformation of a host with epithelial features by c-MYC and the zinc finger protein GKLF. Cell Growth Differ 10: 423-434, 1999.

28. Liu Z, Teng L, Bailey SK, Frost AR, Bland KI, LoBuglio AF, Ruppert JM and Lobo-Ruppert SM: Epithelial transformation by KLF4 requires Notch1 but not canonical Notch1 signaling. Cancer Biol Ther 8: 1840-1851, 2009.

29. Pandya AY, Talley LI, Frost AR, Fitzgerald TJ, Trivedi V, Chakravarthy M, Chhieng DC, Grizzle WE, Engler JA, Krontiras $\mathrm{H}$, et al: Nuclear localization of KLF4 is associated with an aggressive phenotype in early-stage breast cancer. Clin Cancer Res 10: 2709-2719, 2004.

30. Parasramka MA, Dashwood WM, Wang R, Saeed HH, Williams DE, Ho E and Dashwood RH: A role for low-abundance miRNAs in colon cancer: The miR-206/Krüppel-like factor 4 (KLF4) axis. Clin Epigenetics 4: 16, 2012.

31. Sassen S, Miska EA and Caldas C: MicroRNA: Implications for cancer. Virchows Arch 452: 1-10, 2008.

32. Hwang HW and Mendell JT: MicroRNAs in cell proliferation, cell death, and tumorigenesis. Br J Cancer 96 (Suppl): R40-R44, 2007.
33. Shen Y, Tang D, Yao R, Wang M, Wang Y, Yao Y, Li X and Zhang H: microRNA expression profiles associated with survival, disease progression, and response to gefitinib in completely resected non-small-cell lung cancer with EGFR mutation. Med Oncol 30: 750-757, 2013.

34. Yi B, Piazza GA, Su X and Xi Y: MicroRNA and cancer chemoprevention. Cancer Prev Res 6: 401-409, 2013.

35. Shi ZC, Chu XR, Wu YG, Wu JH, Lu CW, Lü RX, Ding MC and Mao NF: MicroRNA-375 functions as a tumor suppressor in osteosarcoma by targeting PIK3CA. Tumour Biol 36: 8579-8584, 2015.

36. Liu Y, Xing R, Zhang X, Dong W, Zhang J, Yan Z, Li W, Cui J and $\mathrm{Lu} \mathrm{Y:}$ miR-375 targets the p53 gene to regulate cellular response to ionizing radiation and etoposide in gastric cancer cells. DNA Repair 12: 741-750, 2013

37. Luo J, Wu J, Li Z, Qin H, Wang B, Wong TS, Yang W, Fu QL and Lei W: miR-375 suppresses IGF1R expression and contributes to inhibition of cell progression in laryngeal squamous cell carcinoma. Biomed Res Int 2014: 374598, 2014.

38. Dong CG, Wu WK, Feng SY, Wang XJ, Shao JF and Qiao J: Co-inhibition of microRNA-10b and microRNA-21 exerts synergistic inhibition on the proliferation and invasion of human glioma cells. Int J Oncol 41: 1005-1012, 2012.

39. Noguchi S, Yasui Y, Iwasaki J, Kumazaki M, Yamada N, Naito S and Akao Y: Replacement treatment with microRNA-143 and -145 induces synergistic inhibition of the growth of human bladder cancer cells by regulating PI3K/Akt and MAPK signaling pathways. Cancer Lett 328: 353-361, 2013.

40. Tiwari N, Meyer-Schaller N, Arnold P, Antoniadis H, Pachkov M, van Nimwegen E and Christofori G: Klf4 is a transcriptional regulator of genes critical for EMT, including Jnk1 (Mapk8), PLoS One 8: e57329, 2013.

41. Zheng X, Li A, Zhao L, Zhou T, Shen Q, Cui Q and Qin X: Key role of microRNA-15a in the KLF4 suppressions of proliferation and angiogenesis in endothelial and vascular smooth muscle cells. Biochem Biophys Res Commun 437: 625-631, 2013.

42. Suer I, Karatas OF, Yuceturk B, Yilmaz M, Guven G, Buge O, Cansiz $\mathrm{H}$ and Ozen M: Characterization of stem-like cells directly isolated from freshly resected laryngeal squamous cell carcinoma specimens. Curr Stem Cell Res Ther 9: 347-353, 2014

43. Lin CC, Sharma SB, Farrugia MK, McLaughlin SL, Ice RJ, Loskutov YV, Pugacheva EN, Brundage KM, Chen D and Ruppert JM: Kruppel-like factor 4 signals through microRNA-206 to promote tumor initiation and cell survival. Oncogenesis 4: e155, 2015.

44. Zhang W, Chen X, Kato Y, Evans PM, Yuan S, Yang J, Rychahou PG, Yang VW, He X, Evers BM, et al: Novel cross talk of Kruppel-like factor 4 and beta-catenin regulates normal intestinal homeostasis and tumor repression. Mol Cell Biol 26: 2055-2064, 2006.

45. Greco A, De Virgilio A, Rizzo MI, Pandolfi F, Rosati D and de Vincentiis M: The prognostic role of E-cadherin and $\beta$-catenin overexpression in laryngeal squamous cell carcinoma. Laryngoscope 126: E148-E155, 2016.

46. Wang Y, Huang C, Reddy Chintagari N, Bhaskaran M, Weng T, Guo Y, Xiao X and Liu L: miR-375 regulates rat alveolar epithelial cell trans-differentiation by inhibiting Wnt/ $\beta$-catenin pathway. Nucleic Acids Res 41: 3833-3844, 2013.

47. Ladeiro Y, Couchy G, Balabaud C, Bioulac-Sage P, Pelletier L, Rebouissou S and Zucman-Rossi J: MicroRNA profiling in hepatocellular tumors is associated with clinical features and oncogene/tumor suppressor gene mutations. Hepatology 47: 1955-1963, 2008.

48. Bracken CP, Khew-Goodall Y and Goodall GJ: Network-based approaches to understand the roles of miR-200 and other microRNAs in cancer. Cancer Res 75: 2594-2599, 2015. 